\title{
Mechanisms of Alphafetoprotein Transfer in the Perfused Human Placental Cotyledon from Uncomplicated Pregnancy
}

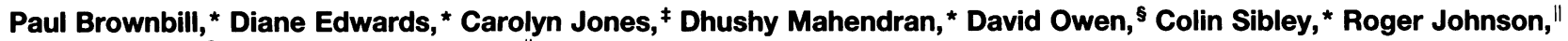 \\ Paul Swanson," and D. Michael Nelson" \\ *Department of Child Health and School of Biological Sciences, St. Mary's Hospital University of Manchester, Manchester M13 OJH \\ United Kingdom; ${ }^{\ddagger}$ Department of Pathological Sciences, University of Manchester, Manchester, M13 9PL United Kingdom; \\ ${ }^{8}$ Radioimmunoassay Laboratory, St. Mary's Hospital, Manchester M13 OJH United Kingdom; "Department of Obstetrics and \\ Gynecology; and 'Department of Pathology, Washington University School of Medicine, St. Louis, Missouri 63110
}

\begin{abstract}
We investigated the mechanisms of alphafetoprotein (AFP) transfer across the human placenta by correlating measurements of AFP transfer with cytochemical localization of AFP. Placental cotyledons were dually perfused in vitro with either the fetal or maternal perfusate containing umbilical cord plasma as a source of AFP. Steady state AFP clearance, corrected for release of endogenous AFP, was $0.973 \pm 0.292$ $\mu \mathrm{l} / \mathrm{min}$ per gram in the fetal to maternal direction $(n=10)$, significantly higher $(P<0.02)$ than that in the maternal to fetal direction $(n=5 ; 0.022 \pm 0.013 \mu \mathrm{l} / \mathrm{min}$ per gram). Clearance of a similarly sized protein, horseradish peroxidase was also asymmetric but clearance of the small tracer creatinine was not. Using a monoclonal antibody, we localized AFP to fibrinoid deposits in regions of villi with discontinuities of the syncytiotrophoblast, to cytotrophoblast cells in these deposits, to syncytiotrophoblast on some villi, and to trophoblast cells in the decidua. We conclude that AFP transfer in the placenta is asymmetric and that there are two available pathways for AFP transfer: $(a)$ from the fetal circulation into the villous core and across fibrinoid deposits at discontinuities in the villous syncytiotrophoblast to enter the maternal circulation; and (b) AFP present in the decidua could enter vessels that traverse the basal plate. $(J$. Clin. Invest. 1995. 96:2220-2226.) Key words: protein • transport $\bullet$ in vitro $\cdot$ placenta $\cdot$ fetus
\end{abstract}

\section{Introduction}

Measurement of the maternal serum alphafetoprotein (MSAFP) ${ }^{1}$ concentration in the second trimester is a common

A preliminary report of this work was presented at a meeting of the Society for Gynecologic Investigation, 24-28 March, 1994, in Chicago, IL.

Address correspondence to Colin P. Sibley, Ph.D., Department of Child Health, University of Manchester, St. Mary's Hospital, Hathersage Road, Manchester M13 OJH, United Kingdom. Phone: 161-276-6484; FAX: 161-224-1013.

Received for publication 19 July 1994 and accepted in revised form 27 July 1995.

1. Abbreviations used in this paper. AFP, alphafetoprotein; HRP, horseradish peroxidase; MSAFP, maternal serum AFP; $\mathrm{K}$, unidirectional clearance.

J. Clin. Invest.

(C) The American Society for Clinical Investigation, Inc.

0021-9738/95/11/2220/07 \$2.00

Volume 96, November 1995, 2220-2226 component of pregnancy care. Elevated MSAFP levels were originally used to screen for fetal neural tube defects. However, recent data indicate that in the absence of fetal anomalies a high MSAFP concentration in the mid-trimester is associated with intrauterine growth retardation, preeclampsia, and increased perinatal mortality (1). Increased fetal to maternal transfer of alphafetoprotein (AFP) across the placenta due to increased placental permeability is often implicated as an etiology for the higher MSAFP level where there is no fetal anomaly. However, this assumption has not been tested directly since the mechanisms involved in AFP transfer in normal pregnancy are unknown.

There are four anatomical barriers for proteins passing between the maternal and fetal circulations of the human placenta: the syncytiotrophoblast which is bathed by maternal blood in the intervillous space, the trophoblastic and capillary basement membranes, and the fetal capillary endothelium. Gitlin (2) suggested that placental transfer of AFP is by diffusion. Both in vivo and in vitro studies have shown that the permeability of the human placenta to inert, hydrophilic tracers is proportional to their coefficients of free diffusion in water (3-9) showing that transfer by paracellular diffusion can occur for a molecule such as AFP. The anatomical correlate for the paracellular route of diffusion in the human placental villus is controversial since the syncytiotrophoblast is a true syncytium. However, we reported that discontinuities in the syncytiotrophoblast layer were present on placental villi from uncomplicated pregnancies and were associated with fibrin containing fibrinoid deposits (10). This observation allowed us to identify these discontinuities as one paracellular route for diffusion of hydrophilic molecules from the maternal to fetal circulation.

The aim of the present study was to investigate the mechanisms of AFP transfer across placental villi from uncomplicated pregnancies. We correlated quantitative measurements of AFP transfer between the fetal and maternal circulations in the dually perfused human placental cotyledon with cytochemical localization of AFP immunoreactivity in sections of perfused tissue. This also allowed us to test the hypothesis that areas of syncytial discontinuity on the villous surface provide one route for AFP transfer between the fetal and maternal circulations.

As the data suggested that AFP clearance in the fetal to maternal direction is greater than that in the maternal to fetal direction, we also measured the fetal to maternal clearance of the similarly sized (molecular radius 3-4 nm) protein horseradish peroxidase (HRP). This allowed comparison to our previous data on maternal to fetal HRP clearance (9) and determination of whether the asymmetry in clearances was a phenomenon affecting all macromolecules.

\section{Methods}

This study was approved by the Central Manchester Health Authority Ethical Committee. 
The placentas from uncomplicated term pregnancies were obtained after vaginal delivery $(n=16)$ or cesarean section $(n=9)$ from women whose MSAFP concentrations were less than two multiples of the median when measured during the second trimester. Cotyledons ( $42.4 \pm 4.3$ grams; mean \pm SEM) were perfused by the method of Schneider, Panigel, and Dancis (11) as adapted in our laboratory (9). The intervillous space and the fetal circulation were perfused at a mean rate of $14.1 \pm 0.1$ and $5.6 \pm 0.1 \mathrm{ml} / \mathrm{min}$, respectively; perfusate recovery for both the fetal and maternal circulations was required to be $>95 \%$ of inflow volume to proceed with the experimental protocol; in the experiments reported here actual perfusate recovery was not significantly different from $100 \%$. Perfusate was initially Earle's bicarbonate buffer containing $5.6 \mathrm{mM}$ glucose, $0.5 \mathrm{mM}$ dextran 70 (mol wt 70-80 kD; Sigma Chemical Co., Poole, United Kingdom) and $0.017 \mathrm{mM}$ bovine serum albumin (Sigma Chemical Co.), equilibrated with $95 \% \mathrm{O}_{2} / 5 \% \mathrm{CO}_{2}$ attaining a pH of 7.4 after gassing. Perfusion with this standard medium was continued throughout the experimental period in three preparations. In the other experiments, a 15-20-min period of dual perfusion with the medium was followed by ongoing perfusion with fresh medium added to either the maternal circulation (in experiments where maternal to fetal transfer was measured) or fetal circulation (in experiments where fetal to maternal transfer was measured). The fresh medium was Earle's bicarbonate buffer containing $5.6 \mathrm{mM}$ glucose, $4.4 \mathrm{mM}$ creatinine (as a small molecular weight extracellular tracer), and either 20 or $50 \%$ (vol/vol) umbilical cord plasma, obtained from other placentas, as a source of AFP. Perfusion was continued on the donor circulation with the medium containing AFP in closed circuit for 40-60 min while the acceptor circulation was perfused with the standard medium without creatinine or AFP in open circuit. Samples of donor and acceptor circulation perfusates were taken at 10 - and 5-min intervals, respectively. At the end of the transfer experiment, cotyledons were perfusion fixed through the fetal circulation with a fixative of $2.0 \%$ formaldehyde $(\mathrm{wt} / \mathrm{vol}$ ) and $2.5 \%$ glutaraldehyde ( $\mathrm{vol} / \mathrm{vol}$ ) in $0.1 \mathrm{M}$ cacodylate buffer at $\mathrm{pH}$ 7.2. The fixed cotyledon was excised, weighed, thinly sliced, immersed in the same fixative for $1 \mathrm{~h}$, and washed in three changes of $0.1 \mathrm{M}$ cacodylate buffer with $0.25 \mathrm{M}$ sucrose at $\mathrm{pH}$ 7.2. The fixed tissue was washed in three changes of $0.1 \mathrm{M}$ Tris hydrochloride buffer at $\mathrm{pH} 7.4$ and stored in this buffer at $4^{\circ} \mathrm{C}$ until processed for histologic examination.

In some experiments HRP (type II; Sigma Chemical Co.) was added to the donor side perfusate containing cord plasma, as described above to a final activity of $17,500 \mathrm{U} /$ liter and in other experiments HRP was added to the standard medium (i.e., without cord plasma) and perfused through the donor side; these experiments were then continued as described above.

Analysis of perfusate samples. The AFP concentrations in perfusate samples were measured by the RIA used for clinical samples at St. Mary's Hospital (12). This RIA uses a well characterized antibody which is unreactive with other serum proteins; the assay has a sensitivity of $5 \mathrm{ng} / \mathrm{ml}$ and a coefficient of variation of $<10 \%$ at the analyte ranges used. Because blood is incompletely removed from the fetal circulation of perfused cotyledons (13), there was release of AFP endogenous to the tissue. We measured this endogenous AFP output into the fetal and maternal perfusates in the three preparations dually perfused with the Earle's salt solution without added cord plasma. These data were used to correct for release of endogenous AFP in experiments where AFP was added to one of the perfusates, as detailed further in Results.

HRP was assayed as previously described $(9,14)$. During the course of this study we found that effluent perfusate contained endogenous peroxidase activity, most likely due to hemoglobin in the samples. However, in the absence of added HRP, the absorbance of perfusate samples at $414 \mathrm{~nm}$ (a wavelength at which HRP at the concentrations used here does not absorb) was linearly correlated $\left(r^{2}=0.99\right)$ with the endogenous peroxidase activity. Therefore, in experiments with added HRP, measurement of absorbance of perfusate effluent samples at $414 \mathrm{~nm}$, together with the standard curve (endogenous peroxidase activity versus absorbance at $414 \mathrm{~nm}$ ), allowed us to correct data for endogenous peroxidase activity. In our previous study of maternal to fetal transfer of HRP (9) we had not been aware of the endogenous peroxidase activity so the clearances reported are therefore an overestimate of the true maternal to fetal HRP clearance. However, we had stored frozen all the samples from that study so that we were able to reanalyze them and correct for the endogenous peroxidase activity.

Creatinine concentrations were measured using Sigma Kit No. 555A (Sigma Chemical Co.). Transfer of AFP, HRP, and creatinine was calculated in terms of unidirectional clearance $(\mathrm{K})$ :

\section{$\mathrm{K}=([$ acceptor side $] \cdot \mathrm{Q}) /([$ donor side $] \cdot \mathrm{W}) \mu \mathrm{l} / \mathrm{min}$ per gram placenta}

where $\mathrm{Q}$ is the measured flow rate in the acceptor circulation and $\mathrm{W}$ is the wet weight of the perfused cotyledon before fixation $(9,15)$.

Data are mean \pm SEM. The Student's $t$ test for paired or unpaired data as appropriate was used for statistical analysis of normally distributed data. The Mann Whitney $U$ test was used for analysis of data which was not normally distributed. A probability of $P<0.05$ was accepted as significant.

Morphological investigations. The histologic evaluation of perfused tissues was effected in two steps. The perfused tissue was examined initially to verify the absence of tissue damage. Villous tissue was diced into fragments about $2-3 \mathrm{~mm}^{3}$ in size, dehydrated in alcohol, cleared in propylene oxide, and infiltrated in a propylene oxide/resin mixture before embedding in Taab epoxy resin (Taab Laboratories Equipment Ltd., Aldermaston, United Kingdom with polymerization at $60^{\circ} \mathrm{C}$ for $72 \mathrm{~h}$. Sections $0.5-\mu \mathrm{m}$ thick were cut on a ultramicrotome (OMUIII; Reichert Scientific Instruments, Buffalo, NY), mounted on glass slides, and stained with $1 \%$ toluidine blue in $1 \%$ borax. Three blocks were examined from each of the upper, middle, and basal areas of the perfused cotyledons. Only those specimens showing minimal trophoblast vacuolation and little artefactual disruption of the syncytiotrophoblast were further processed. Clearance data from the tissue rejected by these criteria were not included in the final analysis.

Tissues fulfilling the above morphological criteria were further processed for cytochemical localization of AFP. 5- $\mu \mathrm{m}$ sections of paraffin embedded specimens were dewaxed in an organic solvent (Americlear; American Scientific, McGaw Park, IL) and absolute ethanol, and endogenous peroxidase was quenched by immersion in $0.6 \%(\mathrm{vol} / \mathrm{vol})$ methanolic peroxide solution for 30 min. Slides were rehydrated in graded ethanols and distilled water and placed in PBS at $\mathrm{pH} 7.4$ for $10 \mathrm{~min}$. A monoclonal antibody to AFP (Clone AFP 1; Serotec Ltd., Kidlington, United Kingdom) was diluted 1:1,000 ( $\mathrm{vol} / \mathrm{vol})$ with PBS and applied to sections for incubation in moisture chambers for $18 \mathrm{~h}$ at $4^{\circ} \mathrm{C}$. Slides were developed using the avidin-biotin-peroxidase complex method (16). A chromogenic precipitate was obtained by immersing sections in $3,3^{\prime}$-diaminobenzidine tetrahydrochloride $(0.25 \mathrm{mg} / \mathrm{ml}$ with $0.003 \%$ hydrogen peroxide; Sigma Chemical Co.) for up to $10 \mathrm{~min}$ and the reaction product was enhanced by brief exposure to $0.125 \%$ osmium tetroxide. Slides were counterstained in Harris' hematoxylin, dehydrated, and coverslipped in a synthetic mounting medium.

Negative controls were placental sections processed identically except with substitution of anti-AFP antiserum by either nonimmune mouse ascites fluid (as a protein-rich source) or McCoy's solution. Staining sections of a human yolk sac germ cell tumor known to express AFP immunoreactivity provided a positive control. Duplicate sets of placental sections from each experiment were compared to these positive and negative control slides by an observer blinded to the treatment conditions. The presence or absence of AFP immunoreactivity was systematically evaluated in the syncytiotrophoblast, the endothelium and connective tissue of the villous core, the fibrin containing fibrinoid deposits, and the decidua, when the plane of section happened to include this area of the cotyledon.

\section{Results}

General. 17 of 27 preparations used in clearance experiments which fulfilled our perfusate recovery criteria also fulfilled our morphological criteria. 5 of the 17 were used to study maternal to fetal AFP clearances and one was used to measure maternal to fetal HRP clearance. Of the remaining 11,10 were used to study fetal to maternal AFP clearances, six of which were also 


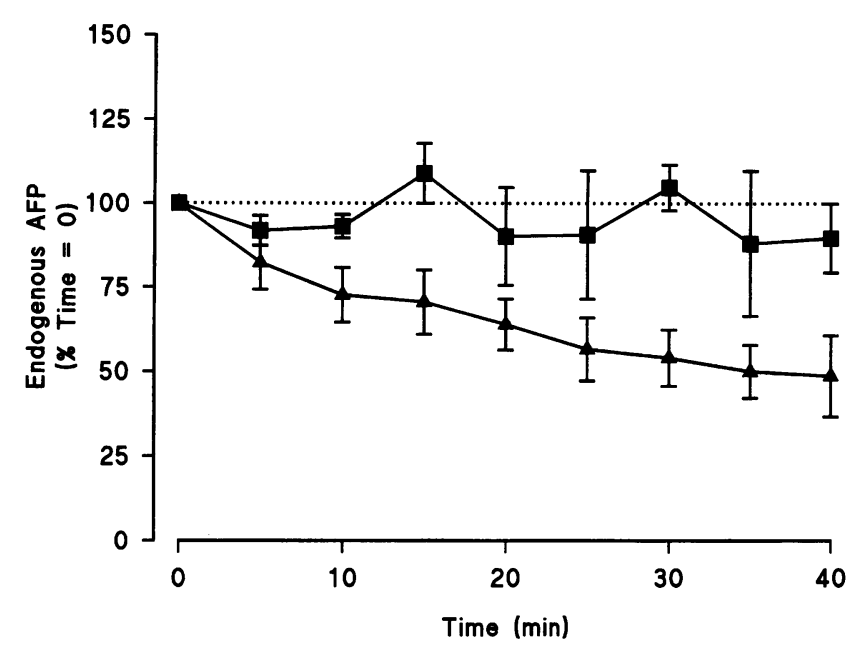

Figure 1. Endogenous AFP output. Cotyledons were perfused with Earle's bicarbonate buffer containing $5.6 \mathrm{mM}$ glucose, $0.5 \mathrm{mM}$ dextran 70 , and $0.017 \mathrm{mM}$ BSA. AFP in maternal $(\mathbf{\Lambda})$ and fetal $(\boldsymbol{\square})$ perfusates was measured by RIA and calculated as a percentage of that in the perfusate at time 0 (i.e., $15-20 \mathrm{~min}$ after the start of dual perfusion). Mean \pm SEM, $n=3$.

used to study fetal to maternal HRP clearances, and one experiment was used to study fetal to maternal HRP clearance alone. Creatinine clearances were measured simultaneously with those of AFP.

After correction for tubing pressure, mean inflow pressure at $40 \mathrm{~min}$ was $38 \pm 6 \mathrm{mmHg}$ on the maternal side and $46 \pm 5$ $\mathrm{mmHg}$ on the fetal side.

$A F P, H R P$, and creatinine clearances. Blood present in the perfused cotyledons is incompletely removed (14), and thus, we noted a continuous elution of AFP into the perfusates throughout the perfusion time period studied when cord plasma was not added to the perfusion medium. This release of endogenous AFP is shown in Fig. 1, where AFP concentrations in perfusate samples are illustrated as a percentage of the AFP concentration in the perfusate measured at time zero (i.e., 15$20 \mathrm{~min}$ after establishing dual perfusion of the fetal vasculature and maternal intervillous space). Endogenous AFP release into the fetal circulation was relatively constant over the time course of the experiments whereas endogenous AFP release from the maternal circulation slowly decreased. In each experiment where AFP was to be added to either the maternal or fetal perfusate, we measured the AFP concentration in the time zero sample obtained before perfusion with the medium containing cord plasma. This AFP concentration in the time zero perfusate and the data shown in Fig. 1 was used to estimate the contribution of endogenous AFP to total AFP measured in each acceptor circulation sample subsequently collected.

Clearance data for AFP are illustrated without correction for endogenous AFP release in Fig. $2 a$ and with correction for endogenous AFP release in Fig. $2 b$. Donor and acceptor side perfusate AFP concentrations at steady state $(40 \mathrm{~min}$; see below) in experiments where fetal to maternal clearance was measured were $59.6 \pm 7.1$ and $0.048 \pm 0.021 \mu \mathrm{g} / \mathrm{ml}$, respectively, and, in experiments where maternal to fetal clearance was measured, $65.1 \pm 9.1$ and $0.085 \pm 0.045 \mu \mathrm{g} / \mathrm{ml}$, respectively. Fetal to maternal clearance increased significantly $(P<0.05$, paired $t$ test) over the first $25 \mathrm{~min}$ and then reached a steady state; the steady state clearance, $\mathrm{K}$, at $40 \mathrm{~min}$ was $0.973 \pm 0.292 \mu \mathrm{l} / \mathrm{min}$

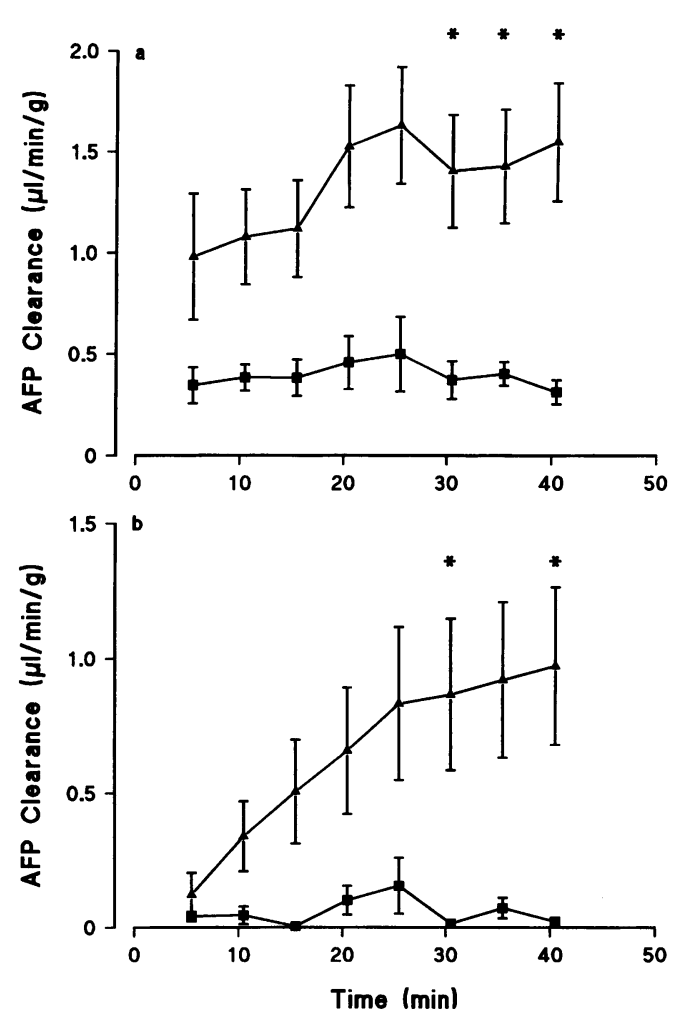

Figure 2. AFP clearance uncorrected ( $a$ ) for release of endogenous AFP into the perfusate and corrected $(b)$ for endogenous AFP release. Cotyledons were initially perfused with Earle's bicarbonate buffer containing $5.6 \mathrm{mM}$ glucose, $0.5 \mathrm{mM}$ dextran 70 , and $0.017 \mathrm{mM}$ BSA. After 15-20 min of dual perfusion Earle's bicarbonate buffer containing 5.6 $\mathrm{mM}$ glucose, $4.4 \mathrm{mM}$ creatinine, and either 20 or $50 \%$ (vol/vol ) umbilical cord plasma was perfused through the maternal circulation (in experiments where maternal to fetal clearance was measured; $\boldsymbol{0})$ or through the fetal circulation (in experiments where fetal to maternal clearance was measured; $\Delta$ ) while the standard perfusate without cord plasma was maintained through the opposite circulation. AFP was measured in timed samples using an RIA and clearance calculated. Mean \pm SEM; $n=5$ for maternal-fetal clearance and $n=10$ for fetal-maternal clearance; $* P$ $<0.02$ vs maternal-fetal clearance (Mann Whitney U test).

per gram placenta when corrected for endogenous AFP. In contrast, maternal to fetal AFP clearance showed minimal change over the time course studied. When corrected for endogenous AFP, maternal to fetal AFP clearance $(\mathrm{K}=0.022 \pm 0.013 \mu \mathrm{l} /$ min per gram placenta) was not significantly different from zero (Fig. $2 b$ ). Fetal to maternal AFP clearance was significantly ( $P$ $<0.02$, Mann-Whitney $U$ test) higher than maternal to fetal AFP clearance at steady state $(40 \mathrm{~min})$ whether or not the correction for endogenous AFP was made (Fig. 2).

Fetal to maternal HRP clearance, corrected for endogenous peroxidase activity, was very similar to that for AFP in this direction and, at steady state $(40 \mathrm{~min})$ was $0.783 \pm 0.232 \mu \mathrm{l} /$ min per gram placenta $(n=7)$. For comparison we reanalyzed samples from our previous study of maternal to fetal clearance of HRP (9), so that endogenous peroxidase activity was corrected for and added to these values data from one further measurement of maternal to fetal HRP clearance, carried out as part of the present study to ensure reproducibility. The maternal to fetal HRP clearance calculated from these data was similar to that for AFP in the same direction; at $40 \mathrm{~min}$ the mean maternal to fetal HRP clearance was $0.103 \pm 0.056 \mu \mathrm{l} / \mathrm{min}$ per 


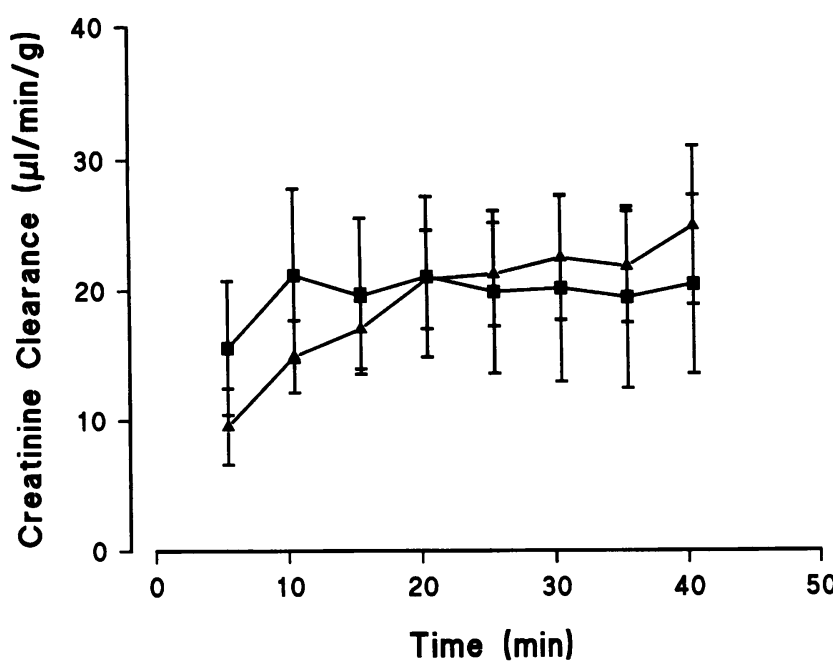

Figure 3. Creatinine clearance. Cotyledons were initially perfused with Earle's bicarbonate buffer containing $5.6 \mathrm{mM}$ glucose, $0.5 \mathrm{mM}$ dextran 70 , and $0.017 \mathrm{mM}$ BSA. After 15-20 minutes of dual perfusion Earle's bicarbonate buffer containing $5.6 \mathrm{mM}$ glucose, $4.4 \mathrm{mM}$ creatinine, and either 20 or $50 \%$ ( $\mathrm{vol} / \mathrm{vol}$ ) umbilical cord plasma was perfused through the maternal circulation (in experiments where maternal to fetal clearance was measured; $\square$ ) or through the fetal circulation (in experiments where fetal to maternal clearance was measured; $\Delta$ ) while the standard perfusate without cord plasma was maintained through the opposite circulation. Creatinine was measured using a colorimetric assay and clearance calculated. Mean $\pm \mathrm{SEM} ; n=5$ for maternal-fetal clearance and $n=10$ for fetal-maternal clearance.

gram placenta $(n=10)$, significantly $(P<0.05$; Mann Whitney $\mathrm{U}$ test) lower than that for HRP in the fetal to maternal direction.

Creatinine clearance rose significantly (paired $t$ test) over the first $10 \mathrm{~min}$ of experimental perfusion and then reached a steady state (Fig. 3). By contrast to AFP and HRP, there was no significant difference for the maternal to fetal creatinine clearance $(\mathrm{K}=20.4 \pm 6.9 \mu \mathrm{l} / \mathrm{min}$ per gram placenta $)$ compared to the fetal to maternal creatinine clearance $(K=24.9 \pm 6.1 \mu \mathrm{l} /$ min per gram placenta).

Immunocytochemistry. We used a monoclonal antibody and peroxidase cytochemistry to localize AFP immunoreactivity in sections of villous tissue from the perfused cotyledons. No immunoreactivity was observed in negative control sections of placenta where nonimmune mouse ascites fluid, rich in proteins, or McCoy's solution were substituted for the AFP antibody (Fig. $4 \mathrm{~A}$ ). The expected pattern of immunoreactivity for AFP was present in nests of cells in positive control sections of a yolk sac germ tumor known to express AFP (Fig. $4 B$ ). The qualitative localization of immunoreactive AFP was identical in placental tissues perfused with or without medium containing cord plasma as a source of AFP, although specimens perfused with the AFP containing cord serum did tend to have more brown staining in the matrix components of the fibrin-containing deposits and the basal plate. Specific AFP immunoreactivity was present in cytotrophoblast cells associated with fibrinoid deposits (Fig. $4 C$ ) and in the decidua (Fig. 4, $D$ and $E$ ) identified when the basal plate of the perfused cotyledon happened to be included in the tissue section. The AFP immunoreactivity was additionally localized to noncellular components of some, but not all, of the fibrinoid deposits (Fig. $4 F$ ) and to the syncytiotrophoblast of some, but not all, placental villi (Fig. $4 C$ and $G$ ). The villous core matrix and associated connective tissue cells, the trophoblast and capillary basement membranes, and the fetal vessel endothelium showed no specific immunoreactivity for AFP (Fig. $4 F$ ). The lumina of fetal vessels occasionally contained peroxidase reaction product, either secondary to endogenous AFP or to incompletely quenched peroxidase activity of red blood cell hemoglobin.

\section{Discussion}

This study is the first to directly investigate mechanisms of AFP transfer across the human placenta from uncomplicated pregnancies. The AFP clearance data show that the rate of AFP transfer from the fetal to the maternal circulation across the placental villus is faster than the AFP transfer rate from the maternal to fetal circulation. This asymmetric clearance of AFP contrasts with the clearance of the small hydrophilic molecule creatinine which was bidirectional and symmetrical. The cytochemical findings indicate that at least two anatomical pathways associated with the placenta are available for AFP entry into the maternal circulation: the AFP leaving fetal vessels can pass through the villous core and across the fibrinoid deposits at sites of discontinuity of the syncytiotrophoblast to enter the maternal circulation without passing through the cytoplasm of the syncytiotrophoblast; the AFP present in the decidua basalis can access the maternal circulation by entering the vessels that traverse the basal plate.

The clearance across the placenta due to paracellular diffusion of an inert, hydrophilic, nonelectrolyte solute that is not limited by flow should be symmetrical (15). Creatinine is a tracer with the listed characteristics and creatinine clearance in this study was symmetrical. In contrast, the clearance of AFP was asymmetrical and predominantly unidirectional from fetal to maternal circulations. The need to correct the clearance data for the endogenous AFP release may mask a small amount of AFP transfer from maternal to fetal circulations and yet, whatever the error in actual values, AFP clearance remains significantly higher for transfer from the fetal to maternal circulation, compared to the transfer from the maternal to fetal compartment.

The asymmetrical clearance of AFP suggests that a more complex mechanism exists for transfer of this protein than paracellular diffusion. The most likely explanation for the asymmetry is that the fetal to maternal clearance of AFP was higher than predicted by a paracellular diffusion model because there was bulk flow of perfusate containing AFP from the fetal to the maternal circulation. The data showing that the clearance of HRP, a protein not normally handled by the placenta, is also asymmetric is consistent with this bulk flow hypothesis. A fetal to maternal hydrostatic pressure gradient would drive such bulk flow across the placental villous exchange area. Schneider and colleagues (17) have previously demonstrated this in experiments where, using the perfused human placental cotyledon, increases in fetal venous pressure were shown to significantly increase fetal to maternal clearance of a 70,000 MW dextran, but not that of their small extracellular tracer, $L$-glucose. Although direct measurement of hydrostatic pressures at the level of exchange was not possible, the fetal arterial perfusion pressures were higher than those in the maternal intervillous space in our experiments. We calculate that only $0.054 \mathrm{ml} / \mathrm{min}$ of perfusate would need to be filtered from the fetal to maternal direction to explain the asymmetry in AFP clearances, if we assume negligible paracellular diffusion. Unfortunately, this additional volume is too small to measure in our system, with our 


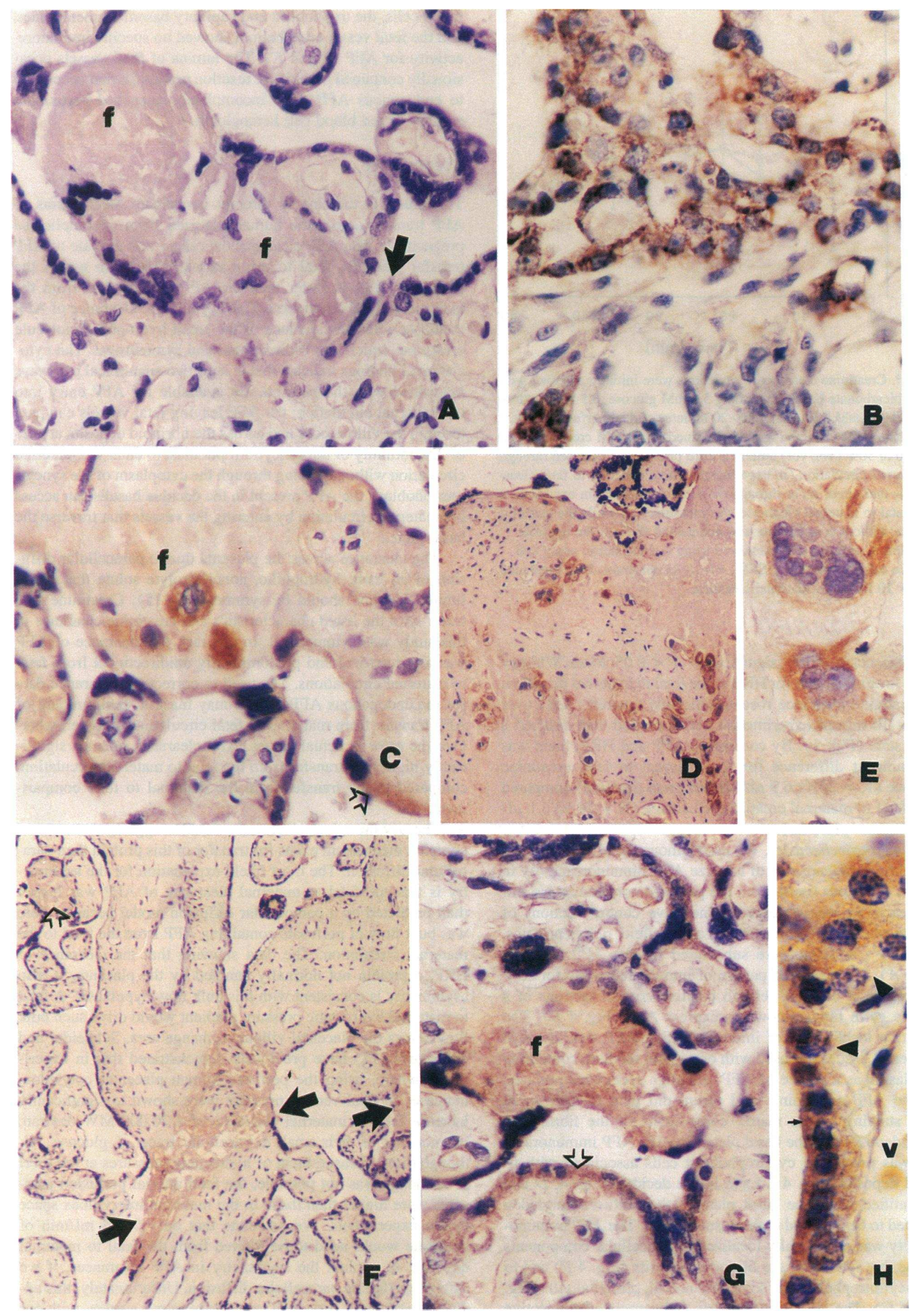


maternal perfusion inflow rate of $14.1 \mathrm{ml} / \mathrm{min}$. Umbilical venous pressure is significantly higher than intervillous space pressure in vitro (18) suggesting that there is a fetal to maternal hydrostatic pressure gradient which could drive bulk flow across the placenta in situ. Štulc and Štulcova (19) provided direct evidence for fetal to maternal bulk flow in vivo across the rat placenta. Bulk flow would not affect creatinine clearance, or that of other extracellular tracers of similar size such as Lglucose, across the human placenta because of the much higher rate of paracellular diffusion for these small molecules. Transcytosis in the fetal to maternal direction across the trophoblast layer offers an additional explanation for the higher fetal to maternal AFP clearance, but the immunolocalization of the AFP largely to the apical cytoplasm of the syncytiotrophoblast does not suggest, but does not exclude, this route.

Fibrin containing fibrinoid deposits occur at discontinuities in the syncytiotrophoblast as a common histologic feature of villi from term human placentas $(10,20)$. The AFP immunoreactivity identified cytochemically in some, but not all, of the deposits in sections of perfused tissue indicate that one available route for paracellular transfer of AFP between the fetal and maternal circulations is through the syncytiotrophoblast layer at the discontinuities in the syncytium. The AFP immunoreactivity of cytotrophoblast in these deposits suggests that AFP uptake, if not synthesis, occurs in these cells and such uptake might modify measured AFP clearance to a small degree. Many deposits did not exhibit immunoreactivity for AFP and no immunoreactivity for AFP was identified in capillary endothelium, villous core connective tissue, or the basement membranes of trophoblast or capillaries. This contrasts with our study of maternal to fetal clearance of horseradish peroxidase, a protein of similar molecular weight to AFP, in the perfused cotyledon model. Cytochemical localization of peroxidase in sections of perfused tissues included not only the fibrinoid deposits but also the trophoblastic basement membrane and villous core macrophages (9). We are therefore uncertain how AFP exiting the fetal circulation gains access to the fibrinoid deposits on the villous surface. The ability to detect AFP cytochemically is limited by the number of AFP molecules per unit area of tissue section and the affinity of the monoclonal antibody for its AFP antigen, among other factors. Fibrinoid contains fibrin and many other serum proteins to which AFP molecules, perhaps nonspe- cifically associate, may allow detection of AFP in this matrix but not other matrices such as the villous core or basement membrane.

An unexpected cytochemical finding suggests a second route for AFP entry into the maternal circulation in vivo. The AFP localized within the decidua would have access to the maternal circulation if this protein molecule can cross into arteries or veins that traverse the basal plate. The immunoreactive AFP in the cytoplasm of trophoblast cells in the decidua could reflect either synthesis or endocytosis of the protein. Synthesis of alphafetoprotein is predominantly by yolk sac and liver during fetal development (2), but Furth and Adinolfi (21) reported some AFP synthesis by placental tissue in vitro. Alternatively, the cytoplasmic immunoreactivity may relate to trophoblast endocytosis of the AFP molecule, as described for other fetal cells (22). Understanding whether the trophoblast either produces or endocytoses AFP is of particular interest in light of recent data associating low maternal serum AFP levels with pregnancies complicated with trisomic fetuses (23). The mere presence of AFP in the basal plate provides a source for maternal serum AFP elevations associated with placenta accreta (24), a pathological pregnancy condition associated with abnormal basal plate anatomy.

The assumption that AFP passes from the fetal to maternal compartments via the amnion or the placenta is implicit in correlating AFP levels with clinical conditions in normal and abnormal pregnancies (1). The mechanism for elevated maternal serum AFP concentrations when abnormal fetal anatomy is present is well described, as this serum protein passes from the fetal circulation across surfaces without skin to enter amniotic fluid and ultimately the maternal circulation. However, the specific mechanisms for AFP transfer in pregnancies without anomalies have not been delineated. In such pregnancies elevated mid-trimester AFP concentrations have been associated with increased placental volume, abnormal basal plate anatomy, chronic villitis, and placental infarction at term (24-26). Our evaluation of AFP transfer in perfused human placental cotyledons from uncomplicated pregnancy not only indicates that the AFP can cross the placental villus, but also offers microscopic mechanisms to explain why enhanced AFP transfer occurs in the presence of gross abnormalities of the placenta. We suggest that the elevated MSAFP levels in some high risk pregnancies

Figure 4. Localization of AFP in sections of perfused placenta. C, D, and E are from specimens perfused without exogenous AFP in the perfusates while $\mathrm{A}, \mathrm{F}, \mathrm{G}$, and $\mathrm{H}$ are from specimens with exogenous AFP added to a perfusate. A monoclonal antibody to AFP and the avidin-biotin-peroxidase technique were used to identify AFP immunoreactivity in tissues. $(A)$ Negative control section of a placental villus processed for cytochemistry but with substitution of nonimmune mouse ascites tumor fluid, rich in proteins, for the specific monoclonal anti-AFP antibody. No background brown staining is present in the fibrinoid deposit $(f)$ located on the villous surface at the discontinuity (arrow) in the syncytiotrophoblast layer. $(B)$ Positive control section from a yolk sac germ cell tumor showing nests of cells with brown staining indicative of specific AFP immunoreactivity. (C) Brown staining localizes AFP immunoreactivity to cytotrophoblasts enmeshed in a fibrinoid deposit $(f)$ and to syncytiotrophoblast on an adjacent villus (arrow). The villous core shows no immunoreactivity. $(D)$ AFP immunoreactivity is apparent as brown staining of trophoblast cells present in this section of the decidua from the basal plate area of a cotyledon perfused without exogenous AFP in the perfusate. An adjacent section to the one shown in D, is illustrated at higher magnification in E and shows two of the trophoblast cells with AFP immunoreactivity in the decidua $(F$ and $G$ ) Lower $(F)$ and higher $(G)$ magnifications of sections of villi previously exposed to fetal perfusate containing AFP. Three fibrinoid deposits (arrows) are present in F with AFP immunoreactivity localized in two of them (closed arrows) and one showing no brown staining (open arrow). The largest fibrinoid deposit bracketed by arrows in the center of $\mathrm{F}$ is located at a junction of two villi where there is a discontinuity in the surface layer of syncytiotrophoblast. The villous core connective tissue and fetal vessels do not show specific AFP immunoreactivity. A higher magnification $(G)$ of a fibrinoid deposit attached to a villus at a discontinuity in the syncytiotrophoblast layer shows the matrix of the deposit is immunoreactive for AFP and an adjacent villus also has syncytiotrophoblast (open arrow) that is immunoreactive for AFP. The villous core shows no specific immunoreactivity. $(H)$ A villus with cytoplasmic immunoreactivity in the syncytiotrophoblast shows more staining in the apical cytoplasm adjacent to the microvillous border ( small arrow) compared to the basal cytoplasm (arrowhead) adjacent to the villous core. A-C, and G $\times 1,520$; $\mathrm{D}$ and $\mathrm{F} \times 600$; $\mathrm{E}$ and $\mathrm{H} \times 3,800$. 
may reflect increased damage to the syncytiotrophoblast of placental villi or abnormal disruption of vessels passing through the basal plate. An increased number of discontinuities in the syncytiotrophoblast would provide more surface area for fetal to maternal AFP passage by either diffusion or filtration, and ultimately higher maternal serum AFP concentration. The AFP molecules in the decidua would have increased access to the maternal circulation if the basal plate anatomy were disrupted, especially if vessels passing through the area were involved. The extent to which irreparable damage to trophoblast or basal plate occurs would determine which pregnancies with elevated mid-trimester AFP levels result in poor pregnancy outcome, including perinatal loss, preeclampsia, or intrauterine growth abnormalities. The capacity of the human placenta to repair itself would explain why most pregnancies with elevated midtrimester AFP concentrations proceed to normal outcomes.

In summary our data show, firstly, that there is asymmetric clearance of AFP across the perfused human placental cotyledon; such asymmetric transfer would be exaggerated in vivo by the considerably higher concentration of AFP in fetal as compared to maternal plasma (2). Secondly, one available route of AFP transfer across the syncytiotrophoblast is across areas of syncytial discontinuity in the villous surface. Finally our data suggest that trophoblasts can alter AFP production and metabolism either by synthesizing or endocytozing this protein.

\section{Acknowledgments}

This work was supported by a North Atlantic Treaty Organization Collaborative Research Grant (NO CRG910105) to C. P. Sibley and D. M. Nelson; by North Western Regional Health Authority Research Infrastructure funding and the Central Manchester Healthcare Trust (Child Health, C. P. Sibley), and by National Institutes of Health grant No. R01 HD29190 (D. M. Nelson)

\section{References}

1. Thomas, R. L., and K. J. Blakemore. 1990. Evaluation of elevations in maternal serum alphafetoprotein: a review. Obstet. Gynecol. Surv. 45:269-283.

2. Gitlin, D. 1975. Normal Biology of $\alpha$-Fetoprotein. Ann. NY Acad. Sci. 259:7-16.

3. Thornburg, K. L., K. J. Burry, A. King-Adams, E. P. Kirk, and J. J. Faber 1988. Permeability of placenta to inulin. Am. J. Obstet. Gynecol. 158:1165-1169.

4. Willis, D. M., J. P. O'Grady, J. J. Faber, and K. L. Thornburg. 1986. Diffusion permeability of cyanocobalamin in human placenta. Am. J. Physiol. 250:R459-R464.

5. Bain, M. D., D. K. Copas, M. J. Landon, and T. E. Stacey. 1988. In vivo permeability of the human placenta to inulin and mannitol. J. Physiol. 399:313319 .
6. Bain, M. D., D. K. Copas, A. Taylor, M. J. Landon, and T. E. Stacey. 1990. Permeability of the human placenta in vivo to four non-metabolized hydrophilic molecules. J. Physiol. 431:505-513.

7. Illsley, N. P., S. Hall, P. Penfold, and T. E. Stacey. 1985. Diffusional permeability of the human placenta. Contr. Gynec. Obstet. 13:92-97.

8. Schneider, H., R. J. Sodha, M. Progler, and M. P. A. Young. 1985. Permeability of the human placenta for hydrophilic substances studied in the isolated dually in vitro perfused lobe. Contrib. Gynecol. Obstet. 13:98-103.

9. Edwards, D., C. J. P. Jones, C. P. Sibley, and D. M. Nelson. 1993. Paracellular permeability pathways in the human placenta: a quantitative and morphological study of maternal fetal transfer of horseradish peroxidase. Placenta. 14:63-74.

10. Nelson, D. M., E. C. Crouch, E. M. Curran, and D. R. Farmer. 1990. Trophoblast interaction with fibrin matrix. Epithelialization of perivillous fibrin deposits as a mechanism for villous repair in the human placenta. Am. J. Pathol. 136:855-865.

11. Schneider, H., M. Panigel, and J. Dancis. 1972. Transfer across the perfused human placenta of antipyrine, sodium, and leucin. Am. J. Obstet. Gynecol. 114:822-828.

12. Khadempour, M. H., I. Laing, and A. H. Gowenlock. 1978. An optimized RIA for maternal serum AFP using polyethylene glycol. Ann. Clin. Biochem. 15:213-219.

13. Barker, G., R. D. H. Boyd, G. H. Lear, and C. P. Sibley. 1987. Incomplete perfusion of the in vitro isolated human placental lobule. J. Physiol. 394:168P. (Abstr.)

14. Berhe, A., W. G. Bardsley, A. Harkes, and C. P. Sibley. 1987. Molecular charge effects on the protein permeability of the guinea-pig placenta. Placenta. $8: 365-380$.

15. Sibley, C. P., and R. D. H. Boyd. 1988. Control of transfer across the mature placenta. In Oxford Reviews of Reproductive Biology Vol. 10. J. R. Clarke, editor. Oxford University Press, Oxford 382-435.

16. Hsu, S.-M, L. Raine, and H. Fanger. 1981. Use of avidin-biotin-peroxidase complex $(\mathrm{ABC})$ in immunoperoxidase techniques; a comparison between $\mathrm{ABC}$ and unlabelled antibody (PAP) procedures. J. Histochem. Cytochem. 29:577580.

17. Schneider, H., J. Štulc, C. Redaelli, and J. Briner. 1988. Effects of elevated umbilical venous pressure on fluid and solute transport across the isolated perfused human placental cotyledon. Trophoblast Res. 3:189-201.

18. Nicolini, U., N. M. Fisk, D. G. Talbert, C. H. Rodeck, N. K. Kochenour, P. Grecko, C. Hubinont, and J. Santolaya. 1989. Intrauterine manometry technique and application to fetal pathology. Prenatal. Diagn. 9:243-254.

19. Štulc, J., and B. Štulcová. 1993. Asymmetrical transfer of inert hydrophilic solutes across the rat placenta. Am. J. Physiol. 265:R670-R675.

20. Fox, H. 1968. Fibrinoid necrosis of placental villi. J. Obstet. Gynaecol. Br. Commonw. 75:448-452.

21. Furth, R. V., and M. Adinolfi. 1969. In vitro synthesis of the foetal $\alpha_{1-}$ globulin in man. Nature (Lond.). 222:1296-1299.

22. Uriel, J. J. 1989. The physiological role of alpha-fetoprotein in cell growth and differentiation. J. Nucl. Med. Allied Sci. 33(Suppl. to \#3):12-17.

23. Greenberg, F., D. Schmidt, A. Darnule, B. Weyland, E. Rose, and E Alpert. 1992. Maternal serum $\alpha$-fetoprotein, $\beta$-human chorionic gonadotropin, and unconjugated estriol levels in midtrimester trisomy 18 pregnancies. Am. J. Obstet. Gynecol. 166:1388-1392.

24. Kupferminc, M. J., R. K. Tamura, T. R. Wigton, R. Glassenberg, and M. L. Socol. 1993. Placenta accreta is associated with elevated maternal serum alpha-fetoprotein. Obstet. \& Gynecol. 82:266-269.

25. Boyd, P. A., and J. W. Keeling. 1986. Raised maternal serum alphafetoprotein in the absence of fetal abnormality placental findings. A quantitative morphometric study. Prenatal. Diagn. 6:369-373.

26. Salafia, C. M., L. Silberman, N. E. Herrera, and M. J. Mahoney. 1988. Placental pathology at term associated with elevated midtrimester maternal serum $\alpha$-fetoprotein concentration. Am. J. Obstet. Gynecol. 158:1064-1066. 